\title{
PREFACE TO THE SPECIAL ISSUE IN HONOR OF PROFESSOR SOJI TSUCHIYA
}

This volume of Laser Chemistry is devoted to honor a distinguished scientist, Professor Soji Tsuchiya, who has conducted pioneering studies in the field of reaction kinetics, reaction dynamics, and molecular spectroscopy and retired from the University of Tokyo in March 1993. Since he organized his own group at the Department of Pure and Applied Sciences of University of Tokyo (Komaba Campus) in 1967, he has been an active supervisor for students, and of course, a world-leading scientist. His research area is so wide that one cannot introduce his prominent achievements in a brief summary, but his major concern has been in the investigation of energy transfer mechanisms in the course of chemical reactions. From the late 60's to the early 70's, he investigated the vibrational energy transfer of vibrationally excited molecules by means of shock waves and infrared and chemical lasers, and reported many valuable results regarding the excitation mechanism of molecules before they pass through the transition state to the product valley. He also investigated the later part of the chemical reaction, i.e. the energy partitioning process among product molecules by the method of infrared chemiluminescence spectroscopy. In 1979, he started a challenging project with Prof. Okitsugu Kajimoto (Kyoto University), Prof. Katsumi Sakurai (University of Tokyo) and others supported by the Grant-in-Aid for Specially Promoted Research from Japanese Ministry of Education. He conducted the projects by combining molecular beam and laser spectroscopic techniques, both of which were regarded as a very new and advanced technique in those days. The first discovery of the Zeeman quantum beat of a polyatomic molecule is one of the noteworthy results from his projects. Until very recently he has been extremely active and he succeeded to clarify the dynamics of molecules in their transition state by using laser spectroscopic techniques such as SEP (stimulated emission pumping) and PHOFEX (photofragment excitation). Soji received the Chemical Society of Japan Award in March 1993 for these original pioneering studies, which gave fundamental insight into molecular energy transfer and chemical reaction dynamics.

On the occasion of his retirement from the University of Tokyo, I planned an international symposium on the molecular energy transfer and reaction dynamics, which have been a central issue for Soji during his long career as a leading scientist. Then, Prof. Okitsugu Kajimoto, Prof. Seiichiro Koda (University of Tokyo), Prof. Katsumi Sakurai, former colleagues of Soji, and Prof. Yukinori Sato (Tohoku University), a former graduate student of Soji, joined me in planning and arranging the symposium. Furthermore, leading physical chemists, Prof. Kinichi Obi (Tokyo In- 
stitute of Technology), Koji Kaya (Keio University), Masahiro Kawasaki (Hokkaido University), Prof. Tamotsu Kondow (University of Tokyo), Dr. Michio Takami (Institute of Physical and Chemical Research), Dr. Harutoshi Takeo (National Institute for Advanced Interdisciplinary Research), Prof. Mitsuo Tasumi (University of Tokyo), Prof. Nobuyuki Nishi (Kyushu University), Prof. Naohiko Mikami (Tohoku University), Prof. Keitaro Yoshihara (Institute for Molecular Science), Dr. Nobuyuki Washida (National Institute for Environmental Studies) supported our project and joined us to form the organizing committee for the international symposium.

The symposium entitled "International Symposium on Molecular Energy Transfer and Reaction Dynamics in Honor of Professor Soji Tsuchiya" was held at the Sanjo Conference Hall on the Hongo Campus of the University of Tokyo during August 20 and 21, 1993, financially supported by Morino Foundation for Molecular Science and Matsuo Foundation and sponsored by The Chemical Society of Japan and The Spectroscopical Society of Japan. On August 20, after an opening address by myself, we had three invited talks; "Thirty Years of Molecular Energy Transfer Studies" by Soji, "Short-time Dynamics Directly from Real and Computed SEP Spectra of Acetylene" by Prof. R. W. Field (Massachusetts Institute of Technology), and "Multiple Laser Probes of Highly Excited States" by Prof. T. R. Rizzo (University of Rochester). On August 21, four invited talks were presented; "Why Quantum Spectra Should Be Assigned by Classical Mechanics" by Prof. E. J. Heller (Harvard University), "Collisional Relaxation and Reactions of Highly Excited Vibrational Levels: A Time-Resolved FT Emission Study" by Prof. H.-L. Dai (University of Pennsylvania), "Solvent-Induced Predissociation of Iodine" by Dr. D. M. Jonas (University of Chicago), and "Dynamics in the Transition State Region for Unimolecular Reactions"' by Prof. C. B. Moore (University of California, Berkeley), and Closing Remarks from Prof. S. Koda. We also had two poster sessions (one for each day) and 39 posters were presented mainly by the domestic participants. In total, we had 151 participants during the two day symposium and valuable and stimulating discussion was enjoyed by the participants in the discussion time after the invited talks and during the poster sessions.

After the symposium, Prof. Koji Kaya, who is on the editorial board of Laser Chemistry, recommended for us to prepare a special issue by calling for papers from the participants of the symposium to commemorate the wonderful scientific interaction arranged on the occasion of honoring Soji Tsuchiya. Two big names in the field of physical chemistry, Prof. Brad Moore and Prof. Bob Field, who both were invited speakers at the symposium, kindly contributed to this volume by sending wonderful articles addressing their interactions with Prof. Soji Tsuchiya.

Professor Soji Tsuchiya is now emeritus professor of the University of Tokyo and professor at Japan Women's University. I sincerely hope that he will continue his research and lead us toward a better understanding of molecular reaction dynamics. As a representative of the organizing committee of the symposium, I would like to express sincere thanks to all the invited speakers and all the participants of the symposium, who made the symposium successful. Thanks are also due to researchers who contributed papers to this special issue and Prof. Koji Kaya who offered an opportunity to publish this issue of Laser Chemistry honoring Professor Soji Tsuchiya. 
Finally, I would like to thank the members of my group, Dr. Charles D. Pibel, Dr. Jun Miyawaki, Dr. Ken Onda, Mr. Mitsuaki Izuha, Ms. Kyoko Ohde, Mr. Tomoki Tasaka, and Mr. Takeki Yazaki, for helping with the running of the symposium.

Kaoru Yamanouchi, Associate Professor Representative of The Organizing Committee of International Symposium on "Molecular Energy Transfer and Reaction Dynamics" Department of Pure and Applied Sciences The University of Tokyo Komaba, Meguroku, Tokyo 153, Japan June 5, 1994 